\title{
START UP AND SATURATION IN MONOTRONS
}

\author{
Joaquim J. Barroso \\ Associated Plasma Laboratory \\ National Institute for Space Research \\ 12201-970 São José dos Campos, SP, Brazil
}

Much emphasis has been placed on microwave generators that do not require an external magnetic field so as to reduce cost, size and weight of the device. One such device is the monotron [1,2], which is a self-excited oscillator consisting of an electron beam that transits across a resonant cavity, which plays the part of an ordinary LCR oscillating tank circuit. The monotron lumped equivalent circuit can be thought of as consisting of a physical tuned circuit connected to a resistor of negative resistance. For transit times near $(\mathrm{N}+1 / 4) \mathrm{T}$, where $\mathrm{N}$ is an integer and $\mathrm{T}$ the oscillation period, electron bunches are formed and upon reaching the cavity's end plate in a decelerating phase of the RF field the bunches transfer energy to the cavity. In the classical monotron configuration [1,2], DC beam power is converted into RF field power, at a maximal 20 percent conversion efficiency. The relation between current and voltage is set by the beam conductance, which is always non linear, so that for one amplitude will there be a given ratio between current and voltage. From the equation of motion of the beam electrons interacting with the $\mathrm{RF}$ fields, such relation - the electronic beam nonlinear conductance $G\left(V^{2}\right)$ -is found by expanding the conversion efficiency up to the tenth power of the RF voltage amplitude. Then using $G\left(V^{2}\right)$ in the energy balance equation (relating the RF power losses in the cavity and the microwave power radiated by the electron beam) we arrive at the van der Pol's equation, just describing the non linear growth and the stationary generation of the electromagnetic mode operating in the cavity driven by the externally injected beam. It is shown that the analytic expressions for the self-excitation condition and for the saturation of the wave growth found on the basis of a 1-D circuit model are in excellent agreement with a full scale $2 \frac{1}{2}$ D particle-in-cell simulation. 